\title{
Enhanced osteoblast adhesion to drug-coated anodized nanotubular titanium surfaces
}

\author{
George E Aninwene II' \\ Chang Yao ${ }^{2}$ \\ Thomas J Webster ${ }^{2}$ \\ 'Department of Biochemical \\ Engineering, University of Maryland, \\ Baltimore, MD; ${ }^{2}$ Division of \\ Engineering, Brown University, \\ Providence, RI, USA
}

\begin{abstract}
Current orthopedic implants have functional lifetimes of only $10-15$ years due to a variety of reasons including infection, extensive inflammation, and overall poor osseointegration (or a lack of prolonged bonding of the implant to juxtaposed bone). To improve properties of titanium for orthopedic applications, this study anodized and subsequently coated titanium with drugs known to reduce infection (penicillin/streptomycin) and inflammation (dexamethasone) using simple physical adsorption and the deposition of such drugs from simulated body fluid (SBF). Results showed improved drug elution from anodized nanotubular titanium when drugs were coated in the presence of SBF for up to 3 days. For the first time, results also showed that the simple physical adsorption of both penicillin/streptomycin and dexamethasone on anodized nanotubular titanium improved osteoblast numbers after 2 days of culture compared to uncoated unanodized titanium. In addition, results showed that depositing such drugs in SBF on anodized titanium was a more efficient method to promote osteoblast numbers compared to physical adsorption for up to 2 days of culture. In addition, osteoblast numbers increased on anodized titanium coated with drugs in SBF for up to 2 days of culture compared to unanodized titanium. In summary, compared to unanodized titanium, this preliminary study provided unexpected evidence of greater osteoblast numbers on anodized titanium coated with either penicillin/streptomycin or dexamethasone using simple physical adsorption or when coated with SBF; results which suggest the need for further research on anodized titanium orthopedic implants possessing drug-eluting nanotubes.
\end{abstract}

Keywords: anodization, titanium, adhesion, simulated body fluid, nanotubes, osteoblasts

\section{Introduction}

The skeletal system is integral for all physical functions in the body. However, many individuals find themselves in a state where one or more of their bones are no longer able to support physiological loading. This may be due to osteoporosis, severe bone damage, bone fracture, or a variety of other osteopathic problems. When bone is irreparably damaged, an implant is required to fasten, support, regrow, or replace existing bone. Titanium and titanium alloys are commonly used as orthopedic implants due to their high mechanical strength, low weight, and bio-inertness. Their bio-inertness is the result of a nonreactive titanium oxide layer that spontaneously forms in the body on the titanium surface. This is beneficial because the oxide layer is biocompatible and keeps the implant from degrading under harsh conditions within the human body (Brunette et al 2000).

Although titanium meets many, if not all, of the requirements to be a successful orthopedic implant material, current titanium implants have long-term failure rates due to a variety of reasons including infection, incomplete acceptance of these foreign materials in the body leading to excessive inflammation, and poor bonding with juxtaposed bone (that is, poor osseointegration). When any synthetic orthopedic implant is placed in the body, a series of immune system responses are initiated, sometimes resulting 
in prolonged soft (instead of hard) tissue encapsulation. This soft tissue encapsulation causes deleterious wear and abrasion. As a result, titanium orthopedic implants have an average lifetime of only about 10-15 years before they have to be replaced (Yao et al 2005).

Decreasing infection, reducing inflammation, and increasing bone growth on titanium surfaces are needed in order to lengthen the lifetime of titanium implants. These events will securely fasten the implant into the juxtaposed bone. There have been many attempts to improve currentlyused titanium for orthopedic applications. It has already been proven that anodizing titanium promotes in vitro bone growth compared to unanodized titanium (Yao et al 2005). If controlled properly, the anodization process creates titania (ie, titanium dioxide) nanotubes on the surface of titanium (the mechanism by which nanotubes form is described elsewhere (Raja et al 2005) and it is briefly shown in Figure 1). These anodized nanotubes increase the roughness of titanium surfaces on the nanoscale and, thus, allow titanium to become more similar to the natural roughness of human bone. Bone-forming cells, or osteoblasts, tend to adhere to surfaces that are similar (both in chemistry and roughness) to natural bone, thus, anodization is a logical biologically-inspired method to promote osteoblast functions (Yao et al 2005).

Moreover, the nanotubes anodized on the titanium surface can serve as reservoirs for drugs small enough to fit inside these tubes. Through anodization, conventional nondrugloaded titanium can be transformed into novel drug-eluting titanium. One can even envision spatial loading of antiinfection, anti-inflammatory, and pro-bone growth drugs to further improve orthopedic implant efficacy. Accordingly, a longer drug release profile can hopefully be established through the use of anodized nanotubular titanium to compensate for the variety of current orthopedic implant failure modes. However, although physical adsorption is the easiest and most direct way to load drugs into porous anodized titanium substrates, there may be surface tension problems that inhibit drug loading. Thus, the specific goals of this in vitro study were to determine drug elution from and osteoblast densities on anodized nanotubular titanium coated with penicillin/streptomycin (anti-infection drugs) and dexamethasone (an anti-inflammatory drug) using physical adsorption compared to a novel manner to load drugs (through the use of simulated body fluid or SBF). Specifically, an alternative to physical adsorption is to simultaneously coat titanium

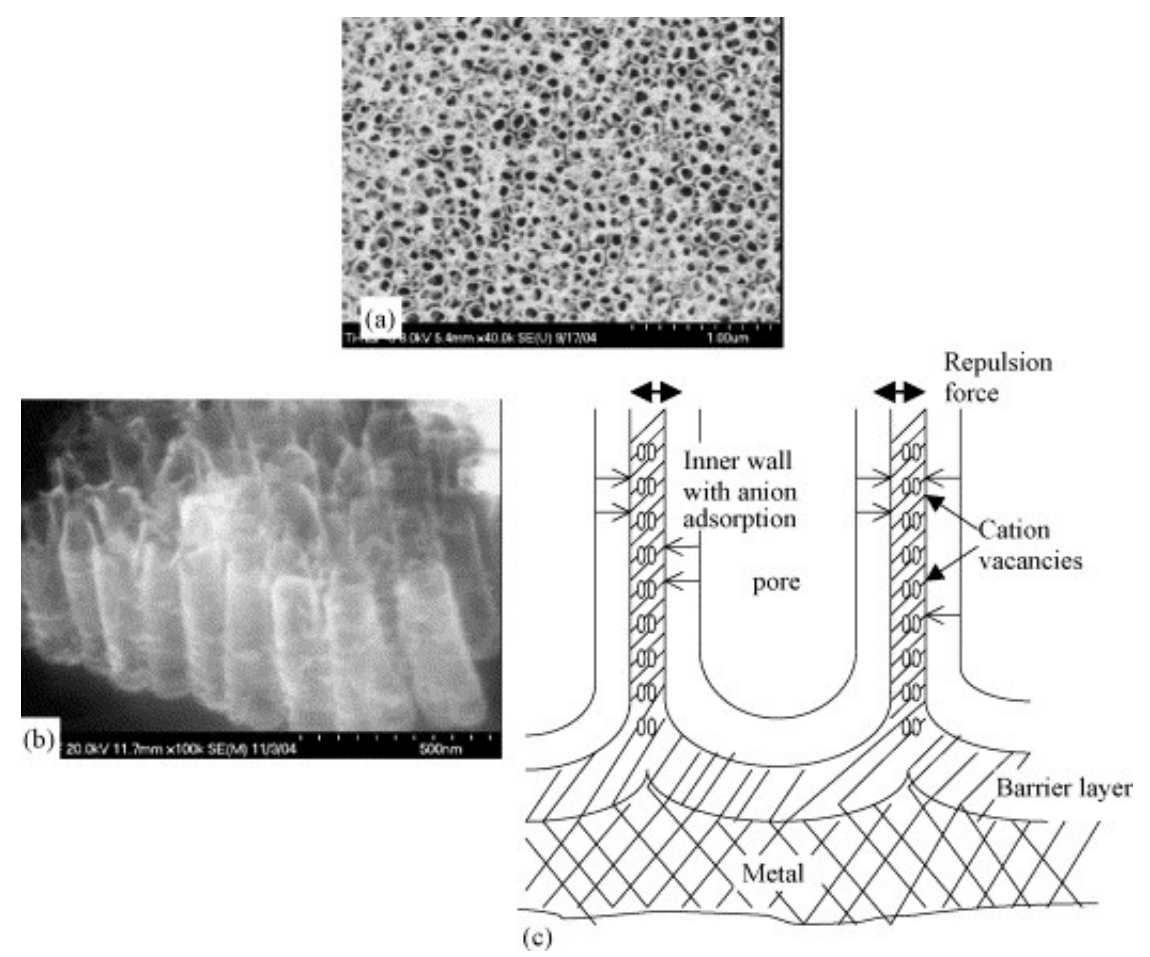

Figure I Separation of nanopores to form an individual nanotubular oxide layer during anodization of titanium. (a) Formation of individual nanotubes during anodization, (b) side view of the nanotubes after anodization and the (c) schematic illustration of the pore separation mechanism. Cation vacancies generated by the dissolution of Ti cations are transported radially from the two sides of the common wall of the neighbor pores. Charges of similar polarity repel and when electrical neutrality is not maintained this repulsion causes separation of pores into individual nanotubes as seen in (b). Copyright @ 2005. Adapted with permission from Raja KS, Misra M, Paramguru K. 2005. Formation of self-ordered nano-tubular structures of anodic oxide layer on titanium. Electrochimica Acta, 5I:I54-65. 
nanotubes with calcium phosphate crystals that form on titanium when soaked in simulated body fluid (SBF) and drugs (Oh et al 2005). Such calcium phosphate crystals may provide optimal surface energy for the efficient coverage of anodized nanotubular titanium with drugs.

\section{Materials and methods}

\section{Titanium anodization}

The anodization procedure followed in this study is shown in Figure 2. Specifically, titanium (99.8\% pure; Alfa Aesar, Ward Hill, MA, USA) was cut to the desired size $(1 \mathrm{~cm} \times$ $1 \mathrm{~cm} \times 2 \mathrm{~mm}$ ). Just prior to anodization, each titanium sample was soaked in a dilute nitric acid and hydrofluoric acid solution for approximately 2 minutes. Titanium was then removed and rinsed with deionized water. Next, the sample was used as an anode in an electrochemical cell, parallel to a platinum mesh acting as the cathode (step 3 in Figure 2). The $1.5 \%$ hydrofluoric acid solution served as an electrolyte solution and completed the circuit. A constant voltage of 20 $\mathrm{V}$ was then applied to the circuit for 10 minutes. After that, the samples were once again rinsed with deionized water.

After the anodization process, titanium was soaked in a $70 \%$ ethanol solution for at least 10 minutes. The submerged samples were then placed in a sonicator (VWR, West Chester, PA, USA; 10W) for 10 minutes, rinsed and then soaked in deionized water for 2 minutes, and dried in an oven. Once dry, titanium was sterilized by autoclaving $\left(120{ }^{\circ} \mathrm{C}\right)$. Unanodized titanium was cleaned and sterilized using the same process.

\section{Drug coating: Simple physical adsorption}

Two approaches were used in this study to coat titanium with drugs: simple physical adsorption and coating with simulated body fluid (SBF). For simple physical adsorption, $120 \mu \mathrm{l}$ of either penicillin/streptomycin (Hyclone, Logan, UT, USA; $5 \mu \mathrm{g} / \mathrm{ml}$ ) or dexamethasone (Sigma, St. Louis, MO, USA; $5 \mu \mathrm{g} / \mathrm{ml}$ ) solutions were placed onto each unanodized or anodized titanium for 3 days at room temperature. The samples were then rinsed with phosphate buffered saline (PBS) to remove excess proteins.

\section{Drug coating: Soaking in simulated body fluid (SBF)}

For the second approach, after titanium cleaning and sterilization as described above, titanium samples were soaked in a 6.0 M sodium hydroxide for approximately 1 hour. The samples were then removed and placed in a furnace at $500{ }^{\circ} \mathrm{C}$ for approximately 2 hours. After this, titanium was allowed to cool to room temperature in air. Titanium was then soaked in a 1.5X SBF (Sigma) solution (controls) or either a mixture of 1.5X SBF and penicillin/streptomycin or dexamethasone for 3 days at room temperature. The same concentrations of

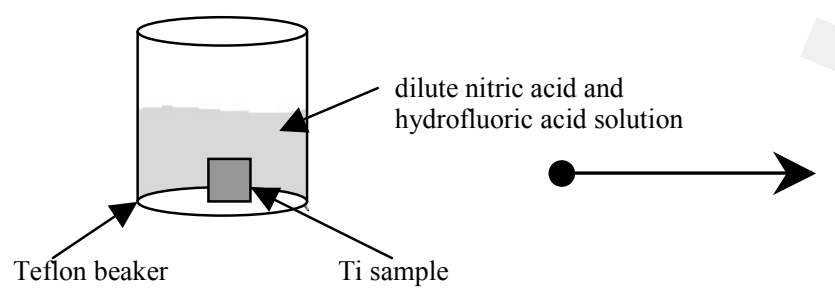

Step 1: Soak in dilute nitric acid and hydrofluoric acid solution for approximately $2 \mathrm{~min}$. This Ti is used as the anode in Step 3.

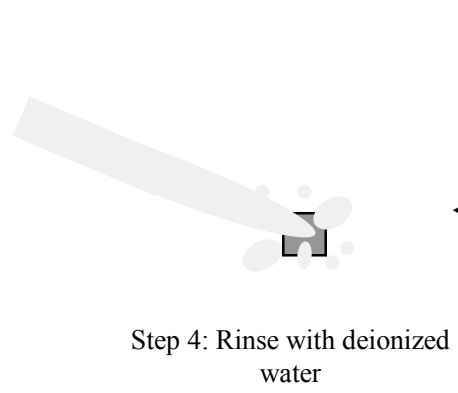
water

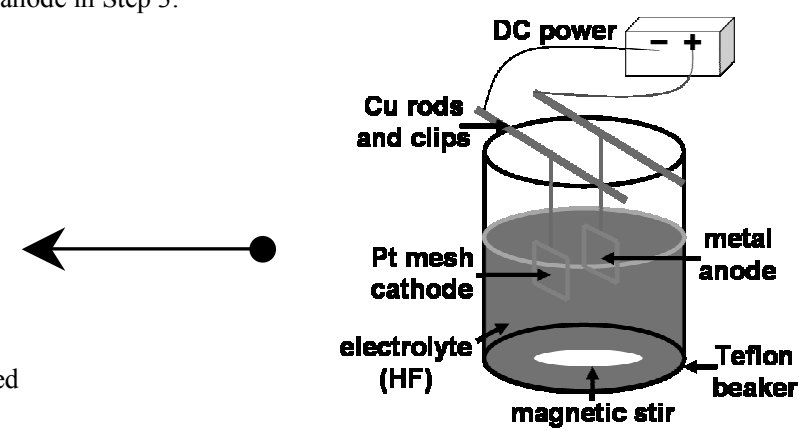

Step 3: Suspend PT cathode and Ti anode in 1.5\%HF solution and apply a voltage of $20 \mathrm{~V}$ for $10 \mathrm{~min}$. This is the Ti from Step 1.

Figure 2 Schematic of the anodization process. 
penicillin/streptomycin and dexamethasone were used in SBF coatings as in the simple physical adsorption method.

\section{Material characterization}

Before and after coating titanium with drugs, they were visualized with scanning electron microscopy according to standard techniques (Yao et al 2005).

\section{Drug elution assay}

To assess the elution of drugs (specifically, penicillin/streptomycin and dexamethasone) into the supernatant solution, substrates were placed in 12 well plates with $2 \mathrm{ml}$ of proteinfree Dulbecco's Modified Eagle's Medium (DMEM; Gibco, Grand Island, NY, USA) under standard cell culture conditions for up to 3 days. After 1, 2, and 3 days, $10 \mu 1$ of the supernatant solution was removed from each well and tested for protein content using the commercially available BCA (Pierce, Rockford, IL, USA) kit and a spectrophotometer. Values were compared to a standard curve to determine specific protein concentration $(\mu \mathrm{g} / \mathrm{ml})$.

\section{Osteoblast density assay}

Osteoblasts (ATCC, USA; CRL 1221) were seeded (3,500 cells $/ \mathrm{cm}^{2}$ ) in DMEM (Gibco) supplemented with $10 \%$ fetal bovine serum (Hyclone) and 1\% penicillin/streptomycin
(Hyclone) onto the substrates of interest to the present study under standard conditions. Osteoblasts were then cultured for up to 2 days. After incubation, the samples were rinsed with PBS to remove nonadherent cells and the adherent cells were fixed using formaldehyde, stained with a DAPI florescent dye (Molecular Probes, Eugen, OR, USA), and counted using fluorescence microscopy.

\section{Statistical analysis}

All experiments were conducted in triplicate and repeated at three different times. Differences between means were assessed using student t-tests at a significance value of $\mathrm{p}<0.1$.

\section{Results}

\section{Material characterization}

As expected, results of this study demonstrated that anodized titanium possessed highly ordered nanotubes whereas unanodized titanium did not (Figure 3); this confirms results from other studies (Yao et al 2005). As observed from the scanning electron microscope images, the average size of the anodized nanotubes in titanium was approximately $60 \mathrm{~nm}$ in diameter. Results of this study also provided evidence that drugs (specifically, penicillin/streptomycin and dexamethasone) were coated

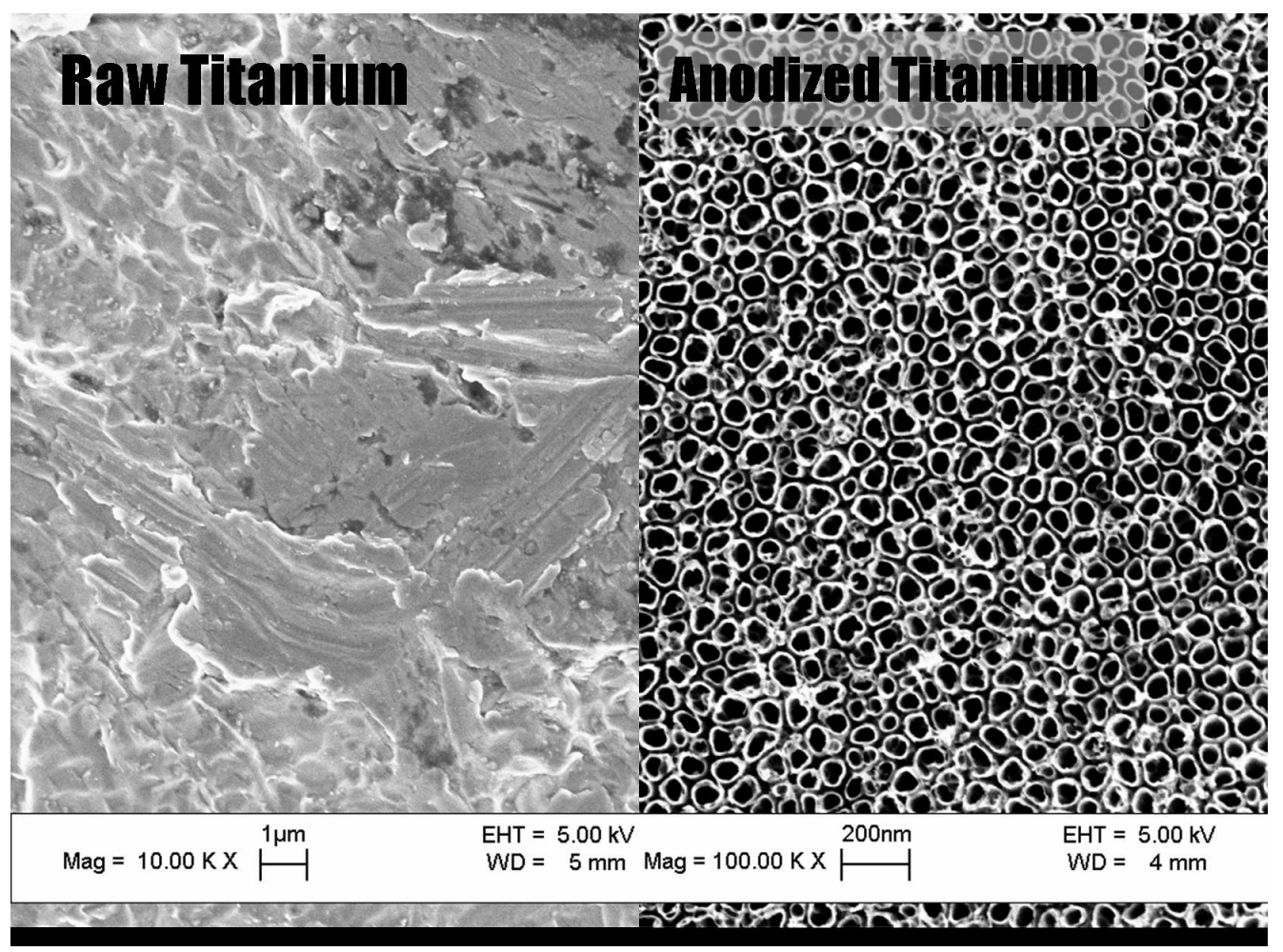

Figure 3 Scanning electron microscope images of unanodized titanium (left) and anodized nanotubular titanium (right). 


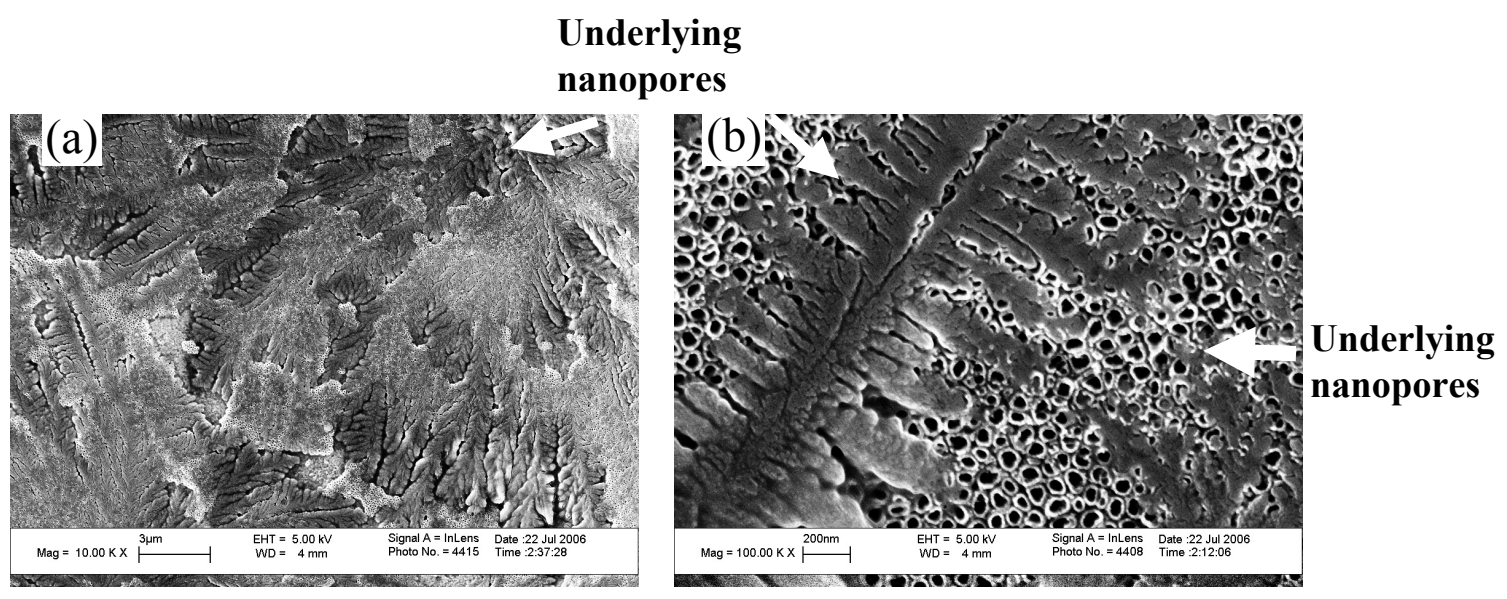

Figure 4 Scanning electron microscope images of penicillin/streptomycin coated on anodized titanium using simple physical adsorption (left: scale bar $=3 \mu \mathrm{m}$; right: scale $\mathrm{bar}=200 \mathrm{~nm}$ ). These images are separate fields of view on the same substrate.

on the titanium substrates after simple physical adsorption (as an example, penicillin/streptomycin is shown adsorbed on anodized nanotubular titanium in Figure 4). In addition, scanning electron micrographs provided evidence of drug coatings in the presence of SBF on titanium substrates (as an example, penicillin/streptomycin is shown adsorbed on anodized titanium in the presence of SBF in Figure 5). Importantly, while the underlying anodized titanium nanopores were visible through the simple physical adsorption of drugs, no underlying nanopores were observed when drugs were coated in the presence of SBF (comparing Figures 4 and 5).

\section{Drug elution}

Results of the present study provided evidence of improved prolonged release of both penicillin/streptomycin and dexamethasone from anodized titanium when coated in the presence of SBF compared to simple physical adsorption (Figure 6). In fact, after 3 days, 3 times more penicillin/ streptomycin was released from anodized titanium coated in the presence of SBF compared to the physical adsorption method. Such results may be related to the previous scanning electron micrographs demonstrating an improved coating of the underlying anodized nanotubes through the use of drugs in SBF compared to simple physical adsorption. In fact, from Figure 6, one can ascertain that in total $2.76 \mu \mathrm{g}$ of penicillin/ streptomycin and $2.3 \mu \mathrm{g}$ of dexamethasone were released from the anodized titanium surfaces when using simple physical adsorption while $3.3 \mu \mathrm{g}$ of penicillin/streptomycin and $2.6 \mu \mathrm{g}$ of dexamethasone were released from the anodized titanium surfaces when coated in the presence of SBF. Thus, the results of the present study suggest that more penicillin/streptomycin and dexamethasone can be incorporated into anodized titanium when coated in the presence of SBF.

\section{Osteoblast adhesion}

Not surprisingly, results of this study confirmed previous studies which have reported greater osteoblast numbers on

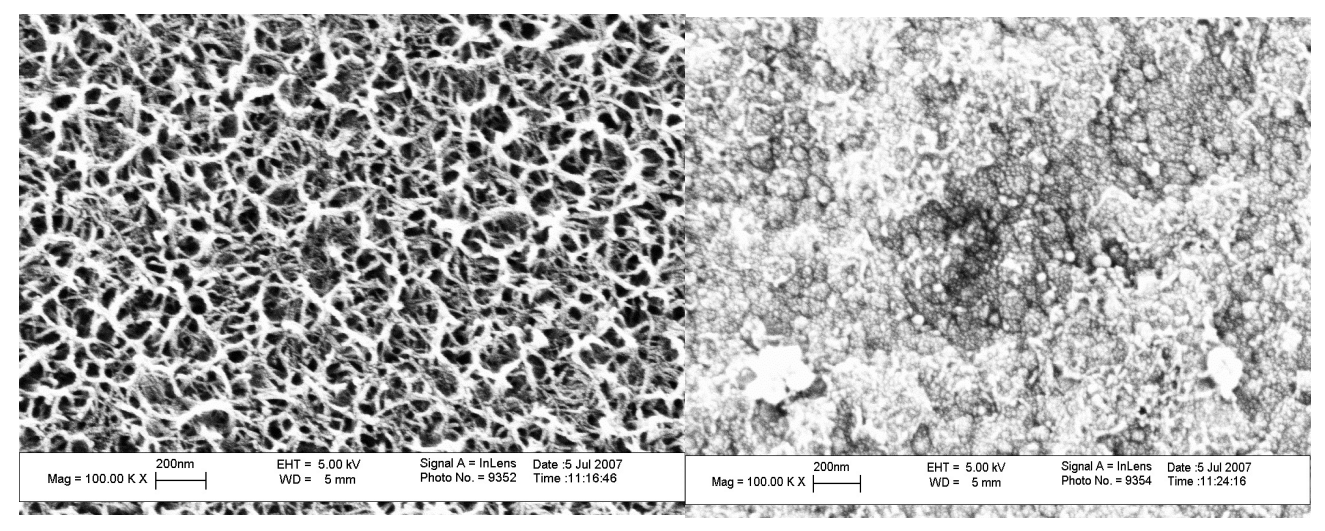

Figure 5 Scanning electron microscope images of anodized titanium alone (left) and when coated with penicillin/streptomycin in simulated body fluid (right). Scale bars $=200 \mathrm{~nm}$. 


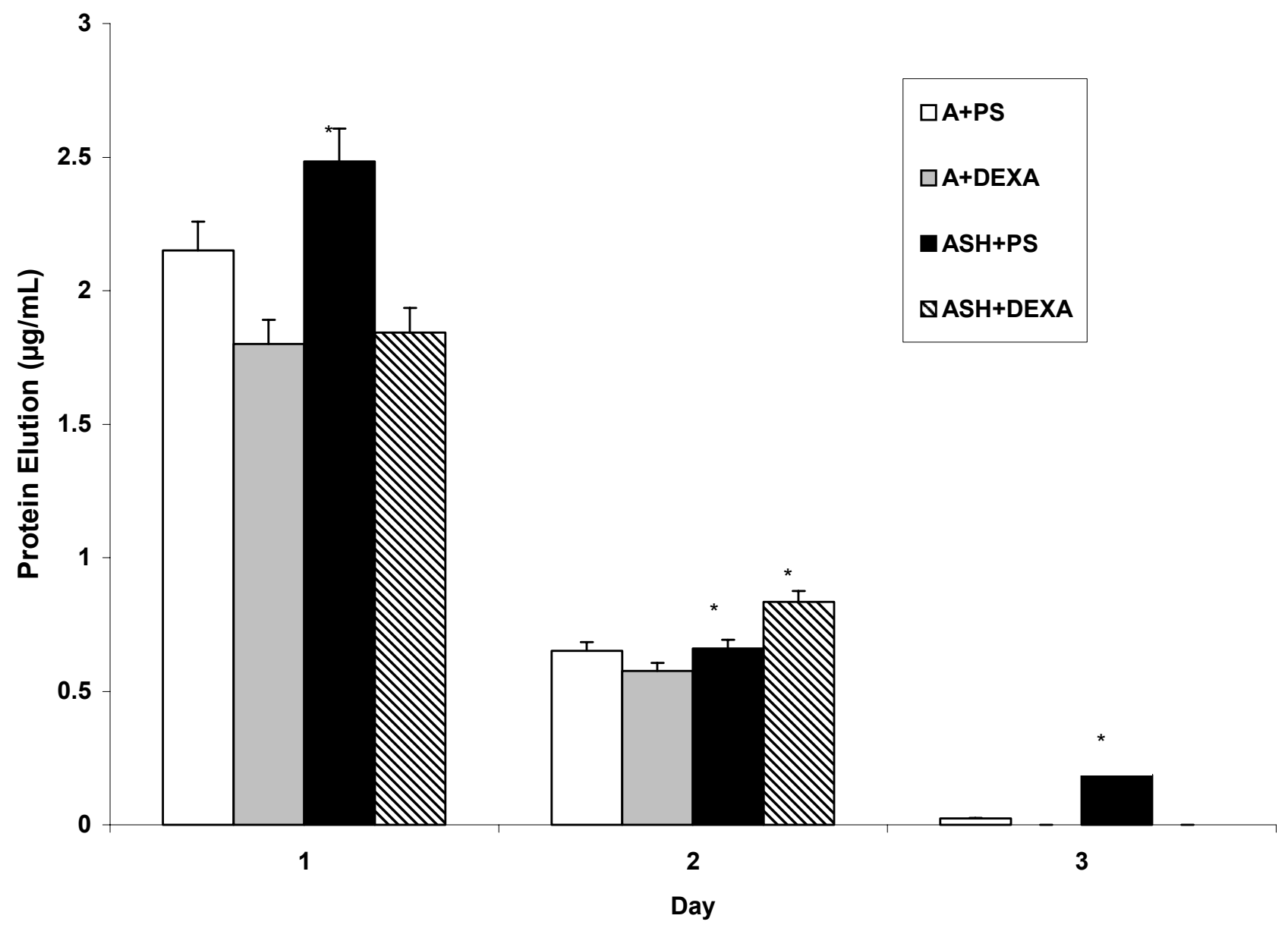

Figure 6 Drug elution from titanium after I, 2, and 3 days.

Abbreviations: A + PS, anodized + penicillin/streptomycin physical adsorption; A + DEXA, anodized + dexamethasone physical adsorption; ASH + PS, anodized, heat-treated and SBF-soaked titanium + penicillin/streptomycin;ASH + DEXA, anodized, heat-treated and SBF-soaked titanium + dexamethasone; SBF, simulated body fluid. Notes: Data $=$ mean $\pm \mathrm{SEM} ; \mathrm{N}=3 ;{ }^{*} \mathrm{p}<0$. I compared to respectively coated drug using physical adsorption.

anodized compared to unanodized titanium (Figure 7) (Yao et al 2005). However, surprisingly, results of the present study also demonstrated improved osteoblast numbers after 2 days of culture on drug-coated anodized titanium samples (whether by simple physical adsorption or through the use of SBF) compared to unanodized titanium. In addition, greater osteoblast numbers where observed on anodized nanotubular titanium coated with drugs in SBF compared to the physical adsorption method. Osteoblast numbers were similar on all substrates of interest after 1 day of culture.

\section{Discussion}

Orthopedic implant failures have been correlated to infection, extensive inflammation, and poor osseointegration into juxtaposed bone. While there have been many efforts to improve osteoblast functions on titanium by modifying its surface properties, little effort has been dedicated to combining drug delivery with current orthopedic implants to fight infection and extensive inflammation. For this reason, titanium which possesses numerous nanotubes may serve as reservoirs for such drugs for their controlled prolonged release. Anodization, under the correct parameters (such as electrolyte solution, voltage, and time) can transform currently-used titanium into titanium which possesses nanotubes. Previous studies have shown that uncoated anodized nanotubular titanium promotes osteoblast functions since it initially adsorbs greater amounts of vitronectin (a key protein that mediates osteoblast adhesion) from serum than unanodized titanium (Yao et al 2005). However, to determine the efficacy of such anodized nanotubular titanium for orthopedic applications involving drug delivery, the objective of this in vitro study was to determine an effective method for loading drugs into anodized titanium and then to determine osteoblast densities on such substrates.

In this manner, physical adsorption is the easiest and most direct way to load drugs into porous anodized titanium 


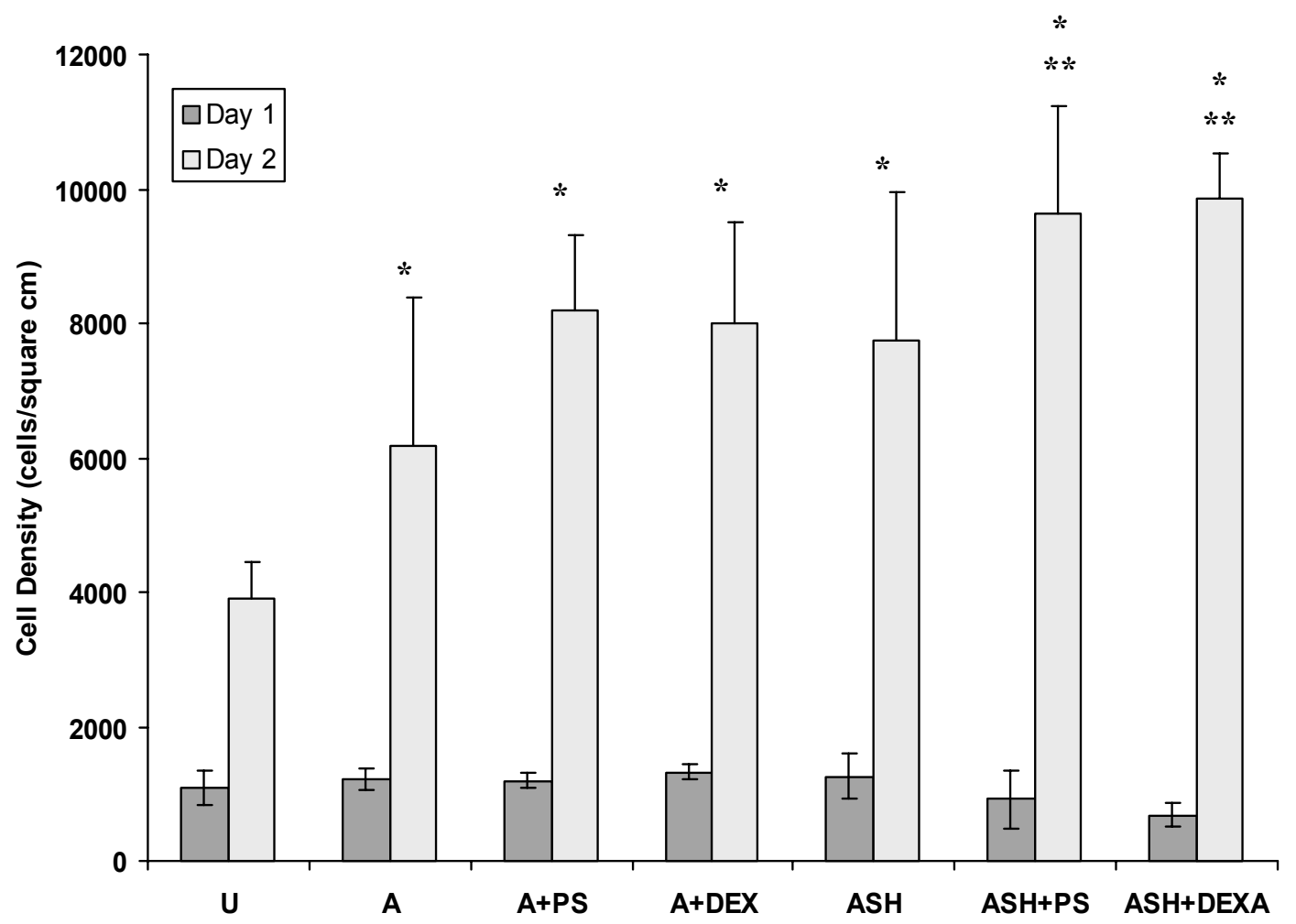

Figure 7 Osteoblast numbers after $\mathrm{I}$ and 2 days of culture.

Abbreviations: $U$, unanodized Ti; A, anodized Ti alone; + + PS, anodized + penicillin/streptomycin physical adsorption; Anodized + DEXA, anodized + dexamethasone physical adsorption; ASH, anodized, heat-treated and SBF-soaked Ti; ASH + PS, ASH + penicillin/streptomycin;ASH + DEXA, ASH + dexamethasone; SBF, simulated body fluid. Notes: Data $=$ Mean $\pm \mathrm{SEM} ; \mathrm{N}=3 ;{ }^{*} \mathrm{p}<0.1$ compared to unanodized titanium; ${ }^{* *} \mathrm{p}<0.1$ compared to respectively coated drug using physical adsorption.

substrates. However, since anodized titanium possesses nanotubes, there may be surface tension problems that inhibit drug loading. For this reason, we investigated an additional manner to load drugs into anodized nanotubular titanium. Specifically, an alternative to physical adsorption is to simultaneously coat titanium nanotubes with calcium phosphate crystals that form on titanium when soaked in SBF and drugs (Oh et al 2005). Such calcium phosphate crystals may provide optimal surface energy for the efficient coverage of anodized nanotubular titanium with drugs. Additional advantages of using SBF during the coating process is that it forms a highly cytocompatible surface for bone cells and its degradation rate can be tailored by varying parameters such as coating thickness. Results from this study provided evidence of a more prolonged release of penicillin/streptomycin and dexamethasone and better osteoblast densities when anodized nanotubular titanium was coated with SBF compared to simple physical adsorption.

Importantly, penicillin/streptomycin and dexamethasone were used as model drugs in this study. Penicillin and streptomycin have long been used as antibacterial drugs. To the best of the authors' knowledge, this is the first time that improved osteoblast functions have been reported when using penicillin/streptomycin drug release. Dexamethasone is a potent member of the glucocorticoid class of steroid hormones and acts as an anti-inflammatory and immunosuppressant. Dexamethasone decreases fibrous tissue formation resulting from excessive foreign body responses, promotes osteoblast differentiation, and potentially increases new bone formation (Mori et al 1999). Since each drug has its limitations, they were used here only as model drugs. It is envisioned that other drugs perhaps with less detrimental side effects and of similar size could also be loaded into the anodized nanotubular titanium to improve orthopedic implant efficacy.

\section{Conclusions}

This study highlighted the initial promise of utilizing anodized nanotubular titanium as a novel drug-eluting orthopedic implant. By using simple physical adsorption of drugs (specifically, penicillin/streptomycin and dexamethasone), improved osteoblast densities were observed compared to unanodized uncoated titanium. Moreover, improved prolonged drug release (up to 3 days) and osteoblast densities 
(up to 2 days) were observed when anodized nanotubular titanium was coated with drugs in the presence of SBF compared to drugs coated using simple physical adsorption. In this manner, although more investigation is needed, this study indicates that anodized nanotubular titanium may hold promise as both a drug eluting orthopedic implant to reduce infection and inflammation while promoting bone growth.

\section{Acknowledgments}

The authors would like to thank the National Science Foundation's Materials Research Science and Engineering Center (MRSEC) at Brown University (DMR 0520651) and the Leadership Alliance for funding. The authors report no other conflicts of interest.

\section{References}

Anselme K. 2000. Osteoblast adhesion on biomaterials. Biomaterials, 21:667-81.
Brunette DM, Tengvall P, Textor M, et al. 2001. Properties and biological significance of natural oxide films on titanium and its alloys. Titanium in Medicine, Springer, 171-230.

Dulgar A. 2007. The importance of hierarchy and the third dimension in bone [online]. Accessed on June 30, 2007. URL: http://www.rpi.edu/ dept/materials/COURSES/NANO/dulgar/nano_index.html

Hoeppner DW, Chandrasekarn V. 1994. Fretting in orthopaedic implants: a review. Wear, 173:189-97.

Long M, Rack HJ. 1998. Titanium alloys in total joint replacement - a materials science perspective. Biomaterials, 19:1621-39.

Mori K, Shioi A, Jono S, et al. 1999. Dexamethasone enhanced in vitro vascular calcification by promoting osteoblastic differentiation of vascular smooth muscle cells. Arterioscler Thromb Vasc Bio, 19:2112-18.

Oh SH, Finõnes RR, Daraio C, et al. 2005. Growth of nano-scale hydroxyapatite using chemically treated titanium oxide nanotubes. Biomaterials, 26:4938-43.

Raja KS, Misra M, Paramguru K. 2005. Formation of self-ordered nanotubular structures of anodic oxide layer on titanium. Electrochimica Acta, 51:154-65.

Yao C, Perla V, McKenzie JL, et al. 2005. Anodized Ti and $\mathrm{Ti}_{6} \mathrm{Al}_{4} \mathrm{~V}$ possessing nanometer surface features enhances osteoblast adhesion. $J$ Biomed Nanotechnol, 1:68-73. 